\title{
Long-term associations between cholinesterase inhibitors and memantine use and health outcomes among patients with Alzheimer's disease
}

\author{
Carolyn W. Zhu ${ }^{\mathrm{a}, *}$, Elayne E. Livote ${ }^{\mathrm{a}}$, Nikolaos Scarmeas ${ }^{\mathrm{a}, \mathrm{c}, \mathrm{g}}$, Marilyn Albert ${ }^{\mathrm{d}}$, Jason Brandt ${ }^{\mathrm{d}}$, \\ Deborah Blacker ${ }^{\mathrm{e}}$, Mary Sano ${ }^{\mathrm{f}}$, Yaakov Stern ${ }^{\mathrm{b}, \mathrm{c}}$ \\ ${ }^{a}$ Department of Geriatrics \& Palliative Medicine, Mount Sinai School of Medicine, New York, NY, USA \\ ${ }^{b}$ Cognitive Neuroscience Division of the Taub Institute for Research in Alzheimer's Disease and the Aging Brain, New York, NY, USA \\ ${ }^{c}$ Gertrude H. Sergievsky Center and the Department of Neurology, Columbia University Medical Center, New York, NY, USA \\ ${ }^{d}$ Department of Psychiatry and Behavioral Sciences, Johns Hopkins University, Baltimore, MD, USA \\ ${ }^{e}$ Department of Psychiatry, Massachusetts General Hospital, Harvard Medical School, Boston, MA, USA \\ ${ }^{f}$ Department of Psychiatry, Mount Sinai School of Medicine, New York, NY, USA \\ ${ }^{g}$ Department of Neurology, Medical School of National and Kapodistrian University of Athens, Athens, Greece
}

\begin{abstract}
Objectives: To examine in an observational study (1) relationships between cholinesterase inhibitors (ChEI) and memantine use, and functional and cognitive end points and mortality in patients with Alzheimer's disease (AD); (2) relationships between other patient characteristics and these clinical end points; and (3) whether effects of the predictors change across time.

Methods: The authors conducted a multicenter, natural history study that included three universitybased AD centers in the United States. A total of 201 patients diagnosed with probable AD with modified Mini-Mental State Examination (MMSE) scores $\geq 30$ at study entry were monitored annually for 6 years. Discrete-time hazard analyses were used to examine relationships between ChEI and memantine use during the previous 6 months reported at each assessment, and time to cognitive (MMSE score $\leq 10$ ) and functional (Blessed Dementia Rating Scale score $\geq 10$ ) end points and mortality. Analyses controlled for clinical characteristics, including baseline cognition, function, and comorbid conditions, and presence of extrapyramidal signs and psychiatric symptoms at each assessment interval. Demographic characteristics included baseline age, sex, education, and living arrangement at each assessment interval.

Results: ChEI use was associated with delayed time in reaching the functional end point and death. Memantine use was associated with delayed time to death. Different patient characteristics were associated with different clinical end points.

Conclusions: Results suggest long-term beneficial effects of ChEI and memantine use on patient outcomes. As for all observational cohort studies, observed relationships should not be interpreted as causal effects.
\end{abstract}

(C) 2013 The Alzheimer's Association. All rights reserved.

\section{Introduction}

Since their introduction, cholinesterase inhibitors (ChEIs) and, later, the N-methyl-D aspartate receptor antag-

\footnotetext{
*Corresponding author. Tel.: + 718-584-9000 ext 6146; Fax: +718-7414211.

E-mail address: carolyn.zhu@mssm.edu
}

onist (memantine) have been shown in short-term clinical trials and longer term open-label extension studies to stabilize or reduce the rate of decline in measures of cognitive function, activities of daily living, and behavior in some patients with Alzheimer's disease (AD) [1-11]. Most rigorous evidence of whether the effects of ChEIs and memantine are sustained over longer periods of time would come from long-duration, prospective, placebo-controlled trials. 
However, such trials are not only costly to conduct, there also are ethical concerns associated with exposing patients to placebo in trials of long duration because ChEIs and memantine have become the standard of care for patients with AD. In the absence of these trials, observational studies based on practice-based populations may be one of the only ways to evaluate the effects of these medications [12].

Several studies have assessed the effects of ChEIs and/or memantine treatment in real-world clinic settings [13-22]. Results from these studies have been mixed. In one of the first observational studies on the effects of ChEI on patient outcomes, Doody and colleagues [13] found slowed decline in cognitive function after a year in patients treated with ChEIs compared with untreated patients. Comparing patients treated with ChEI or ChEI + memantine combination therapy with an untreated earlier cohort of patients, Atri and associates [17] also reported slower decline in cognition and function in the treated group. Persistent treatment has been shown to be associated with slowed decline in cognition and function, but effects may be lost if treatment is disrupted $[3,18]$. On the other hand, in another study comparing a cohort of patients treated with ChEIs with an earlier cohort of untreated patients, Lopez and coworkers [14,19] reported no association between ChEI use and time to cognitive and functional decline or to death, but significant delays in nursing home admission.

In an earlier study using a large, multicenter cohort of patients with probable $\mathrm{AD}$ who were monitored prospectively up to 6 years from early-disease stages, we reported that patterns of ChEI and memantine use changed substantially over time and were consistent with practice guidelines of initiating ChEIs in mild to moderate $\mathrm{AD}$, and adding memantine in moderate to severe $\mathrm{AD}$ [23]. In the current study, we take advantage of the availability of important clinical characteristics (eg, comorbid conditions, psychiatric symptoms) that were not controlled for before, the long follow-up period, and more current data, and further investigated the following questions: Are ChEIs or memantine use associated with length of time to reach cognitive and functional outcomes and death? Are these associations stable over time?

\section{Methods}

\subsection{Sample}

Data are drawn from the Predictors 2 cohort, consisting of patients recruited from Columbia University Medical Center, Johns Hopkins School of Medicine, and Massachusetts General Hospital. The study was approved by each local institutional review board. The inclusion/exclusion criteria have been described fully elsewhere [24-26]. Briefly, subjects met Diagnostic and Statistical Manual of Mental Disorders, edition 3, revised, criteria for primary degenerative dementia of the Alzheimer type and National Institute of Neurological Diseases and Stroke/Alzheimer's
Disease and Related Disorders Association criteria for probable AD. Enrollment required a modified Mini-Mental State Examination score $\geq 30$, equivalent to approximately $\geq 16$ on the Folstein MMSE $[27,28]$. Clinical diagnosis of $\mathrm{AD}$ has been confirmed in $93 \%$ of those with postmortem evaluation [26].

Study recruitment began in 1997, when widespread use of ChEIs began in the United States, and is ongoing. After the baseline assessment, patients were monitored annually. Those who missed a particular visit could respond at a subsequent visit. The cohort used in the current analysis included 201 patients who were monitored for up to 6 years and provided data for 785 visits. Of these 201 patients, 13 had 6 years of follow-up visits, 27 had 5 years of follow-up visits, 37 had 4 years of follow-up visits, 34 had 3 years of followup visits, and 41 had 2 years of follow-up visits. One hundred twenty-three patients $(61 \%)$ did not miss any visits, 15 patients $(7 \%)$ missed one visit, 19 patients $(9 \%)$ missed two visits, 22 patients (10\%) missed three visits, and the rest of the patients missed four or more visits. Median follow-up for the cohort was 4 years (mean, 3.5 years; SD, 2.0).

\subsection{Measures}

\subsubsection{Clinical end points}

We used MMSE scores to assess patients' cognitive status and constructed a dichotomous variable indicating an MMSE score $\leq 10$ at each visit. We used Blessed Dementia Rating Scale (BDRS) Parts I and II (Instrumental and Basic Activities of Daily Living) to assess patients' functional status and constructed a dichotomous variable indicating a BDRS score $\geq 10$ at each visit. We chose these cutoff points because similar scores have been used as outcomes in many studies. Exploratory analyses of neighboring end points (ie, MMSE score $\leq 8$ and BDRS score $\geq 8$ ) did not change estimation results substantively. Patient deaths were most often reported by family members when we attempted to complete follow-up visits. For patients who could not be contacted, information on death was obtained through the National Death Index.

\subsubsection{Main independent variables: ChEI and memantine} use

All prescription and over-the-counter medication use during the previous 6 months were reported at each visit by the patient and informant on a medication acquisition form. Information reported included name of medication, number of days taking the medication, dosage, and number of pills per day. Because ChEIs have been shown to have similar efficacy despite slightly different pharmacological properties, we combined all ChEIs into one group.

Because of the consistency of medication use reported in this sample, we constructed dichotomous variables indicating ChEI and memantine use during the 6 months prior to each assessment as our main independent variables instead 
of using more complex measures that take into account number of days of use or medication dosages.

\subsubsection{Other substantive control variables}

To isolate the effects of ChEI and memantine use, we controlled for the following time-variant variables in the analysis. Columbia University Scale for Psychopathology in $\mathrm{AD}$, a semistructured interview administered by physicians or trained research technicians, was used to measure psychotic symptoms [29]. A modified Unified Parkinson's Disease Rating Scale was used to measure presence of extrapyramidal signs [30,31]. Information on patients' living arrangements at each assessment was dichotomized as living at home or in a long-term care facility. The following time-invariant characteristics were included in the analysis: baseline cognition, function, number of comorbidities, baseline age, sex, education, and study site [26,32]. Because ChEIs and memantine were approved by the U.S. Food and Drug Administration for treatment of $\mathrm{AD}$ at different times, year of study entry was included to control for availability of medications on the market and any differences that could be related to different entry times into the study. Because most of the sample population was white $(\mathrm{n}=188,94 \%)$, ethnicity was not included in the models.

\subsection{Analysis}

We used discrete-time hazard models to examine the relationships between ChEI and memantine use, and time to cognitive and functional end points and mortality. We used binary, time-specific event indicators for BDRS scores, MMSE scores, and death to reflect the first year a patient reached a clinical end point. Right censoring occurred when a patient did not reach a clinical end point by their most recent assessment. Such patients would have a zero on the time-specific event indicator for all assessment intervals up to the most recent assessment. Observations with missing outcomes were dropped from the analysis.

The estimated discrete-time hazard is the conditional probability that patients reach a clinical end point in an assessment interval, given that they did not reach the end point in an earlier interval. Although multiple observations from each patient may be correlated in longitudinal data, this conditional probability can be treated as if it came from a distinct, independent observation, and therefore it is not necessary to account for clustering effects within the individual [33]. For each outcome, we first estimated a set of models that included only time effects (year $=0,1$, $2, \ldots 5)$. This set of models describes for the entire sample the hazard profile for each clinical end point. If the estimated coefficients are approximately the same, the risk of reaching a clinical end point is unrelated to time and the hazard function is flat. If the estimated coefficient increases over time, the risk of reaching a clinical end point increases and the hazard function increases over time. We tested the constant hazard assumption using a likelihood ratio test based on model deviance statistics.

Next, we examined bivariate relationships between each potential substantive covariate (eg, ChEI use) and the clinical end points after controlling for time effects. These main effects models contain the underlying proportionality assumption that each covariate has the same effect on the outcome in every time period. We tested the proportionality assumption of each covariate using deviance statistics obtained from the unconstrained model that included the main effect of the covariate and an interaction term of the covariate and time in the constrained main effects model. If the unconstrained covariate model did not fit better than the main effects model, the covariate did not violate the proportionality assumption and only main effects for that covariate were included in subsequent analyses. If the unconstrained covariate model fit better than the main effects model, both the covariate and its interaction with time were included in subsequent analyses [34].

Last, we estimated a full, multivariate model that included (1) all six binary time-effects indicators, (2) main effects for covariates that did not violate the proportionality assumption, and (3) main effects and interaction with time effects for covariates that violated the proportionality assumption. To account for possible clustering (eg, within sites), robust standard errors were reported. The results of this full model allowed interpretation for each individual covariate effect on our clinical end points after adjusting for all other covariates.

\section{Results}

\subsection{Baseline characteristics}

At baseline, patients' average age was 76 years (SD, 8.1 years), 123 were female (61\%), and $176(88 \%)$ lived at home (Table 1). Patients were highly educated, with an average of 14 years of schooling (SD, 3.1 years). Average MMSE score was 22.0 (SD, 3.4) and average BDRS score was 3.6 (SD, 2.1). Sixty-eight patients had psychotic symptoms (34\%) and 29 had extrapyramidal signs (16\%). Almost half the patients $(\mathrm{n}=96,48.4 \%)$ reported no comorbid conditions, a third $(\mathrm{n}=67,34.1 \%)$ reported one, and the remaining 38 patients (19\%) reported two or more comorbid conditions.

Table 2 presents data on the number patients who were available for observation at each assessment interval, and the number and proportion of those who were taking ChEIs or memantine. Because only patients with mild $\mathrm{AD}$ were included at study entry, four fifths of the 201 patients $(n=161)$ reported using ChEIs at baseline and only $2 \%(\mathrm{n}=4)$ reported using memantine, all of whom used it in combination with ChEIs. By year 6, the proportion of patients who reported using ChEIs decreased slightly to $74.4 \%$, the proportion of patients who reported using memantine increased to $48.8 \%$, and the proportion of patients who reported not 
Table 1

Patient characteristics at baseline $(n=201)$

\begin{tabular}{lll}
\hline Variables & Mean (SD) & Range \\
\hline Age, y & $76.3(8.0)$ & $49-95$ \\
Female, $\%$ & 61.2 & \\
Years of schooling, \% & & \\
$\quad \leq 12$ & 42.3 & \\
$13-15$ & 15.9 & \\
$\quad \geq 16$ & 41.8 & \\
Living in a nursing home, \% & 12.4 & \\
Folstein Mini-Mental State Examination & $21.9(3.4)$ & $12-30$ \\
$\quad$ score & & \\
Modified Mini-Mental State Examination & $37.2(6.5)$ & $14-52$ \\
$\quad$ score & & \\
Blessed Dementia Rating Scale score & $3.6(2.1)$ & $0-10.5$ \\
Presence of psychiatric symptoms, \% & 33.8 & \\
Presence of extrapyramidal signs, \% & 15.9 & $0-8$ \\
No. of comorbidities & $0.8(1.1)$ & \\
Site, $\%$ & & \\
$\quad$ Columbia & 44.3 & \\
Johns Hopkins & 26.9 & \\
$\quad$ Mass General & 28.9 & \\
Year of study entry, \% & & \\
1997-1998 & 13.2 & \\
1999 & 7.7 & \\
2000 & 25.8 & \\
2001 & 25.3 & \\
2002 2007 & 13.2 & \\
\hline
\end{tabular}

Abbreviation: SD, standard deviation.

taking either ChEIs or memantine remained relatively steady. Overall, 182 patients $(91 \%)$ reported taking ChEIs at some point during the study and 19 patients (9.5\%) were never treated with ChEIs. Eighty-one patients (40.2\%) reported taking memantine at some point during the study 120 patients (59.7\%) were never treated with memantine. Patients who were treated with ChEIs at some point were monitored for an average of 3.6 years (SD, 2.0 years) compared with 2.1 years (SD, 1.7 years) for those who

Table 2

Proportion of patients at each visit who reported taking cholinesterase inhibitors or memantine

\begin{tabular}{|c|c|c|c|c|c|c|c|}
\hline \multirow{2}{*}{$\begin{array}{l}\text { Number of patients at } \\
\text { each visit }\end{array}$} & \multicolumn{7}{|l|}{ Year } \\
\hline & 0 & 1 & 2 & 3 & 4 & 5 & 6 \\
\hline Total & 201 & 159 & 127 & 98 & 93 & 66 & 43 \\
\hline \multicolumn{8}{|l|}{ Taking ChEI } \\
\hline No. of patients & 161 & 131 & 99 & 81 & 70 & 48 & 32 \\
\hline Proportion of patients & 80.1 & 82.4 & 78.0 & 82.7 & 75.3 & 72.7 & 74.4 \\
\hline \multicolumn{8}{|l|}{ Taking memantine } \\
\hline No. of patients & 4 & 13 & 19 & 32 & 41 & 34 & 21 \\
\hline Proportion of patients & 2.0 & 8.2 & 15.0 & 32.7 & 44.1 & 51.5 & 48.8 \\
\hline \multicolumn{8}{|c|}{ Taking both ChEI and memantine } \\
\hline No. of patients & 4 & 12 & 16 & 29 & 33 & 30 & 19 \\
\hline Proportion of patients & 2.0 & 7.5 & 12.6 & 29.6 & 35.5 & 45.5 & 44.2 \\
\hline \multicolumn{8}{|c|}{ Did not take either ChEI or memantine } \\
\hline No. of patients & 40 & 27 & 25 & 14 & 15 & 14 & 9 \\
\hline Proportion of patients & 19.9 & 17.0 & 19.7 & 14.3 & 16.1 & 21.2 & 20.9 \\
\hline
\end{tabular}

Abbreviation: ChEI, cholinesterase inhibitor. were never treated with ChEIs. Patients who were treated with memantine at some point were monitored for an average of 4.4 years (SD, 1.5 years) compared with 2.9 years (SD, 2.0 years) for those who were never treated with memantine.

Throughout the study period, the proportion of patients who used specific ChEIs remained relatively stable. During the visits in which patients reported taking ChEIs, $61.7 \%$ reported taking donepezil; 13.6\%, galantamine; and 4.8\%, rivastigmine. We examined in detail the extent and consistency of using individual medications among those who reported taking the medication. Table 3 presents baseline data on the proportion of patients who reported taking each medication every day ( $\geq 180$ days during the previous 6 months) and the proportion of those who took effective dosages for each medication. At baseline, $80.6 \%$ of patients who were taking donepezil reported taking it every day, and $98.3 \%$ of those who were taking donepezil reported taking it at effective doses. During the study period, in $93.3 \%$ of the visits in which patients reported taking donepezil, they also reported that they took it every day during the previous 6 months, and almost all reported taking it at effective doses.

\subsection{Unconditional hazard models}

Figure 1 describes the hazard profile for the entire sample (ie, the conditional probability that an individual would reach a clinical end point given that the individual did not reach the end point in an earlier interval). The hazard function for each outcome increased over time and was most pronounced in patient function. Likelihood ratio tests of the constant hazard assumption was rejected for all outcomes (all $P<.01$ ).

\subsection{Multivariate discrete-time hazard analyses}

Table 4 reports coefficient estimates of final multivariate models that examined time to reach functional and cognitive end points and mortality. Consistent with unconditional hazard models, estimated hazard for each outcome increased significantly over time. Year of study entry and site were not associated significantly with any outcome.

After controlling for patient characteristics, ChEI use was associated with longer time to reaching the functional end point (estimated coefficient, -1.282 ; standard error, 0.433 ; computed odds ratio [OR], 0.278) and mortality (OR, 0.409). Memantine use was associated with longer time to mortality (OR, 0.443). (Full computations of odds ratios are available on request.) Better baseline cognition was associated with longer time to reaching all end points. For each point improvement in baseline MMSE score, estimated odds of reaching the functional end point was $11 \%$ lower, of reaching the cognitive end point was $21 \%$ lower, and of dying was $13 \%$ lower. Better baseline function was also associated with longer time to reaching the functional end point and mortality. A 1-point worsening in baseline 
Table 3

Extent and consistency of cholinesterase inhibitor and memantine use by visit among patients who reported taking the medication

\begin{tabular}{|c|c|c|c|c|}
\hline \multirow[b]{2}{*}{$\begin{array}{l}\text { Generic } \\
\text { name }\end{array}$} & \multicolumn{2}{|c|}{$\begin{array}{l}\text { Prevalence at baseline } \\
\text { visit }\end{array}$} & \multicolumn{2}{|c|}{$\begin{array}{l}\text { Study period prevalence } \\
\text { from all visits }\end{array}$} \\
\hline & $\begin{array}{l}\text { Taking } \\
\text { medication for } \\
180+\text { days, } \%\end{array}$ & $\begin{array}{l}\text { Taking } \\
\text { effective } \\
\text { dose, \%* }\end{array}$ & $\begin{array}{l}\text { Taking } \\
\text { medication for } \\
180+\text { days, } \%\end{array}$ & $\begin{array}{l}\text { Taking } \\
\text { effective } \\
\text { dose, \%* }\end{array}$ \\
\hline Donepezil & 80.6 & 98.3 & 93.3 & 98.9 \\
\hline Galantamine & 83.3 & 61.1 & 88.7 & 73.6 \\
\hline Rivastigmine & 77.8 & 77.8 & 81.1 & 78.9 \\
\hline Memantine & 88.9 & 88.9 & 86.1 & 96.4 \\
\hline
\end{tabular}

NOTE. A total of 201 patients were included at baseline and contributed to 785 observations for the longitudinal sample throughout the study period.

*Effective dose per day for donepezil is defined to be $5 \mathrm{mg}$ or $10 \mathrm{mg}$; for galantamine, 16 to $24 \mathrm{mg}$; rivastigmine, 6 to $12 \mathrm{mg}$; and for memantine, 10 $\mathrm{mg}$ or $20 \mathrm{mg}$.

BDRS score almost doubled the estimated odds of reaching the functional end point $(\mathrm{OR}, 2.0)$. Similarly, a 1-point worsening in baseline BDRS score was associated with $36 \%$ increased odds of dying (OR, 1.36). Over time, the increased risk of reaching the functional end point and mortality from a 1-point increase in baseline BDRS scored attenuated, but the effect was not statistically significant. Compared with patients without extrapyramidal signs, the odds of reaching functional end points for those with extrapyramidal signs was 5.6 times greater, and the odds of dying was 2.3 times higher. At each assessment interval, compared with patients without psychiatric symptoms, the odds of reaching functional end points for those with psychiatric symptoms was significantly higher, with attenuating effects over time.

\subsection{Secondary sensitivity analyses: Exploring potential association between clinical end points and irregular ChEI use}

A small subsample of patients $(n=19)$ in this study reported never having been treated with ChEIs. Compared with patients who were treated with ChEIs at some point during the study, these patients were older, more likely to be female, had less education, had fewer years of followup, and had worse baseline MMSE scores. To test whether our results were sensitive to including patients who were never treated, we reestimated our models using only patients who were treated with ChEIs at some point during the study. Results were substantively similar.

During the long course of the illness, it is likely that patients may change from treated to untreated status or vice versa. The following treatment changes were observed in 56 patients: (1) 26 patients reported taking ChEIs at study entry and later discontinued; (2) 14 patients reported either taking ChEIs at study entry, discontinued at some point, and resumed treatment later, or were not taking ChEIs at study entry, initiated treatment at some point, and discontinued later; and (3) 16 patients initiated and continued ChEI treatment after study enrollment. Because persistent treatment has been associated with slowed decline in cognition and function $[3,18]$, we explored patterns of discontinuation as related to reaching clinical outcomes in these 56 patients. Bivariate distributions between medication use and clinical end points did not show patients were taken off ChEIs as their conditions deteriorated, although power to detect differences was limited. Among the 81 patients who used memantine at some point during the study, the vast majority continued treatment once started, only six patients discontinued after one or two assessments, one of whom reported resuming at a later time.

\section{Discussion}

In this study, we assessed long-term effects of ChEIs and memantine on the natural history of $\mathrm{AD}$ outside the context of controlled trials and open-label extension studies to provide a more real-world perspective. A large cohort of patients from early stages of AD was monitored prospectively for 6 years. After controlling for important clinical characteristics, we found ChEI use was associated with longer time to reaching functional end points and death, and memantine use was associated with delayed mortality. As for all observational cohort studies, common caution in interpreting results is needed. In particular, the effects of $\mathrm{ChEI}$ and memantine use observed in this study should not be interpreted as causal effects.

A small number of studies have examined the relationship between ChEI use and mortality. An early randomized trial on tacrine use reported a trend toward lower mortality among those who received higher doses $(>120 \mathrm{mg} /$ day $)$ in open-label follow up [5]. Another study reported significantly lower 3-year mortality in tacrine users than nonusers [20]. However, a more recent observational study comparing patients on ChEIs with a matched, historical sample of patients who were never exposed to ChEIs reported no association between ChEI use and cognitive and functional status at 1-year of follow-up or 3-year mortality [14]. Another

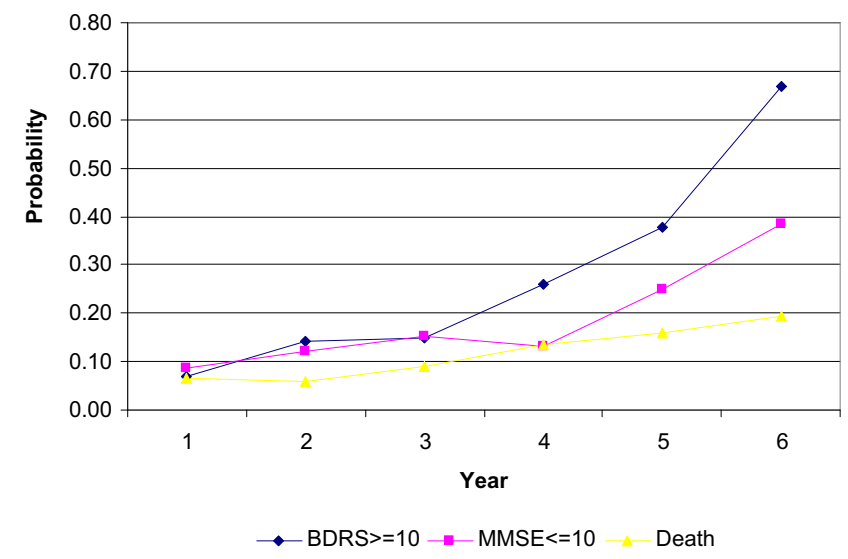

Fig. 1. Unconditional hazard model for functional, cognitive, and mortality end points. BDRS, Blessed Dementia Rating Scale; MMSE, Mini-Mental State Examination. 
Table 4

Multivariate results of discrete-time hazard models of time to reach functional and cognitive endpoints and mortality*

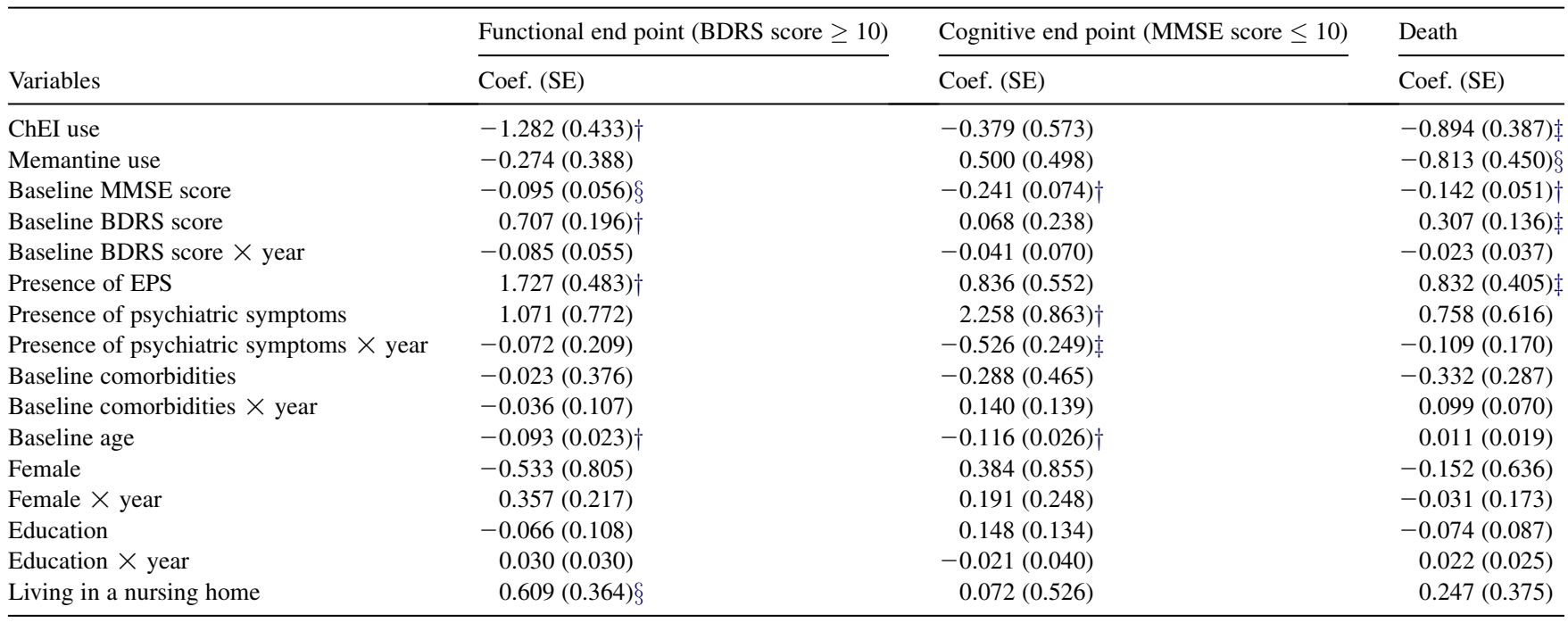

Abbreviations: BDRS, Blessed Dementia Rating Scale; MMSE, Mini-Mental State Examination; Coef., coefficient; SE, standard error; ChEI, cholinesterase inhibitor; EPS, extrapyramidal signs.

*All models controlled for indicators of assessment interval, year of study entry, and site. Robust standard errors are reported.

${ }^{\dagger} P<.01$.

${ }^{\ddagger} P<.05$.

${ }^{\S} P<.10$

study reported ChEI use alone or ChEI + memantine use were not associated with time to death [19].

A direct comparison of our results with these studies is difficult because of differences in study samples and methodologies. Perhaps the closest study to ours is that of Lopez and colleagues [19], in which 443 patients with probable $\mathrm{AD}$ with at least one annual follow-up in a large urban Alzheimer's Disease Research Center were monitored since 1997. Several differences in patient characteristics between these two studies should be noted. First, compared with the Lopez study [19], patients in our sample were, on average, 3 years older (mean age, 76 years vs 73 years), but with higher MMSE scores at study entry (mean MMSE score, 21.9 vs 18.2). It is possible that patients in our sample had an earlier start in ChEI therapy, similar to those who participate in clinical trials, and that this may have led to the stronger mortality effects observed in our study [35-37]. Compared with the study by Lopez and colleagues [19], in which only $3 \%$ of subjects were never treated with ChEIs, $10 \%$ of our patients reported never having been treated with ChEIs. Interestingly, compared with the treated patients, the never-treated group in our study was older whereas they were significantly younger in the study by Lopez and colleagues [19].

It is also important to note methodological differences between these studies. For example, although we included all recruited patients in our analyses, analysis in the study by Lopez and colleagues [19] was restricted to patients with at least one follow-up. Differential rates of decline or differential effectiveness of medications in patients who did not have any follow-up data may have contributed to differences in study results. In several earlier studies, patients who were not treated with ChEIs were derived from historical cohorts that may have confounding effects on the results [14,17]. It is likely that patients in the treated, more recent cohorts were diagnosed earlier than those in the untreated, earlier cohorts. Improvements in medical care in more recent cohorts may have contributed to the relative slowing of disease progression in treated groups. These possibilities should be tested empirically in future studies.

Although estimated associations between ChEI and memantine use and clinical end points are independent of patient characteristics that we controlled for in the models, unobserved characteristics that may differ between groups may remain and may lead to possible biases in our results. It is possible that patients who took these drugs have naturally slower disease progression and their milder disease course was attributed to drug treatment. As in all observational studies, we cannot rule out the possibility of effects influencing observed relationships between treatment and outcomes. It is possible that from frequent, consistent contact with AD center staff, medical management of our patients, particularly those who were treated actively with medications, were better than those without such access or who were untreated, and may have biased our results.

Several studies that examined the effects of $\mathrm{ChEI}+$ memantine combination therapy reported additional beneficial effects of combination therapy in slowed cognitive and functional decline and delayed nursing home placement $[17,19]$. In this study, the vast majority of patients 
who used memantine used it in combination with ChEIs, with memantine monotherapy reported in only 20 visits from 14 patients. Therefore, we could not distinguish the effects of memantine monotherapy vs ChEI + memantine combination therapy. However, the statistically significant effects of both ChEI and memantine suggest beneficial effect of ChEI + memantine combination therapy in delaying mortality.

Our ability to estimate duration of medication use was limited by our data collection method. At each visit, patients were asked about medication use in the previous 6 months; however, follow-up was performed annually. Therefore, medication use was known for half the interval between visits. This loss of information adds noise to estimation models. However, unless there were systematic biases in medication use or discontinuation at 6-month intervals that were not captured in the data, this loss of information should not bias our estimation results.

There are several important strengths of our study. First, there is the large number of well-characterized AD patients who were monitored prospectively from early disease stages and examined by the same measures, in the same memory disorders units, and by the same group of clinicians over multiple years with good follow-up rates. In contrast to AD clinical trial and open-label extension studies that typically include data of, at most, 1 or 2 years, the duration of our study is substantially longer. Second, our study avoids the limitations of most clinical trials, which often have stringent inclusion and exclusion criteria. The sample of patients included in this study and results obtained from these patients therefore reflect more real-world clinical practice. Third, this study addresses several methodological issues in longitudinal analyses. Because data on clinical outcomes are often collected at discrete-time intervals (eg, annually), the commonly used continuous-time methods (eg, Cox models) are limited. We used the more appropriate discrete-time survival models, included both time-invariant and time-variant covariates, and relaxed the often violated proportional hazards assumption when appropriate [33,34,38]. Therefore, our results go beyond findings from short-term clinical trials and provide evidence of the effects of ChEIs and memantine that is fuller and more nuanced, and has real-world clinical relevance in the treatment of patients with $\mathrm{AD}$.

\section{Acknowledgments}

The Predictors Study is supported by federal grant AG07370, with additional support from federal grants, RR00645, and U01AG010483. Dr Sano is supported by the Department of Veterans Affairs, Veterans Health Administration. The views expressed in this article are those of the authors and do not necessarily represent the views of the Department of Veterans Affairs. The authors all certify that they have no conflict of interest to report in this manuscript. The sponsor had no role in the design, methods, sub- ject recruitment, data collections, analysis, and preparation of this article.

\section{RESEARCH IN CONTEXT}

1. Systematic review: Randomized, controlled trials that examine the effects of cholinesterase inhibitors and memantine on patient outcomes over long periods of time are difficult to conduct, and observational studies are limited with mixed results.

2. Interpretation: Our results extend beyond the context of controlled trials and open-label extension studies; our sample has less stringent inclusion/exclusion criteria and is more current, with a substantially longer study duration. Our methodology allows a more nuanced analysis so results have more real-world relevance in the treatment of patients with Alzheimer's disease.

3. Future discussions: Future directions include considering instrumental variables or propensity score approaches to address some study limitations, such as the possible that patients who took cholinesterase inhibitors and memantine have naturally slower disease progression, and their milder disease course was attributed to drug treatment, or the possibility of other variables influencing observed relationships between treatment and outcomes. We will extend our study from our current clinical sample to community samples.

\section{References}

[1] Grimmer T, Kurz A. Effects of cholinesterase inhibitors on behavioural disturbances in Alzheimer's disease: a systematic review. Drugs Aging 2006;23:957-67.

[2] McShane R, Areosa S, Minakaran N. Memantine for dementia. Cochrane Database Syst Rev 2006;2:CD003154.

[3] Burns A, Gauthier S, Perdomo C. Efficacy and safety of donepezil over 3 years: an open-label, multicentre study in patients with Alzheimer's disease. Int J Geriatr Psychiatry 2007;22:806-12.

[4] Birks J. Cholinesterase inhibitors for Alzheimer's disease. Cochrane Database Syst Rev 2009;1:CD005593.

[5] Knopman D, Schneider L, Davis K, Talwalker S, Smith F, Hoover T, et al. Long-term tacrine (Cognex) treatment: effects on nursing home placement and mortality: Tacrine Study Group. Neurology 1996;47:166-77.

[6] Winblad B, Engedal K, Soininen H, Verhey F, Waldemar G, Wimo A, et al. A 1-year, randomized, placebo-controlled study of donepezil in patients with mild to moderate AD. Neurology 2001;57:489-95.

[7] Winblad B, Poritis N. Memantine in severe dementia: results of the 9M-Best Study (benefit and efficacy in severely demented patients during treatment with memantine). Int J Geriatr Psychiatry 1999; $14: 135-46$.

[8] Reisberg B, Doody R, Stoffler A, Schmitt F, Ferris S, Mobius HJ. Memantine in moderate-to-severe Alzheimer's disease. $\mathrm{N}$ Engl J Med 2003;348:1333-41. 
[9] Reisberg B, Doody R, Stoffler A, Schmitt F, Ferris S, Mobius HJ. A 24week open-label extension study of memantine in moderate to severe Alzheimer disease. Arch Neurol 2006;63:49-54.

[10] Farlow M, Anand R, Messina J Jr, Hartman R, Veach J. A 52-week study of the efficacy of rivastigmine in patients with mild to moderately severe Alzheimer's disease. Eur Neurol 2000;44:236-41.

[11] Mohs RC, Doody RS, Morris JC, Ieni JR, Rogers SL, Perdomo CA, et al. A 1-year, placebo-controlled preservation of function survival study of donepezil in AD patients. Neurology 2001;57:481-8.

[12] Karlawish JH, Whitehouse PJ. Is the placebo control obsolete in a world after donepezil and vitamin E? Arch Neurol 1998; 55:1420-4.

[13] Doody RS, Dunn JK, Clark CM, Farlow M, Foster NL, Liao T, et al. Chronic donepezil treatment is associated with slowed cognitive decline in Alzheimer's disease. Dement Geriatr Cogn Disord 2001; 12:295-300.

[14] Lopez OL, Becker JT, Wisniewski S, Saxton J, Kaufer DI, DeKosky ST. Cholinesterase inhibitor treatment alters the natural history of Alzheimer's disease. J Neurol Neurosurg Psychiatry 2002; 72:310-4.

[15] Brodaty H, Woodward M, Boundy K, Barnes N. Naturalistic treatment of Alzheimer's disease with galantamine: 12-month follow-up from the NATURE study. CNS Drugs 2007;21:335-6.

[16] Hansen RA, Gartlehner G, Lohr KN, Kaufer DI. Functional outcomes of drug treatment in Alzheimer's disease: a systematic review and meta-analysis. Drugs Aging 2007;24:155-67.

[17] Atri A, Shaughnessy LW, Locascio JJ, Growdon JH. Long-term course and effectiveness of combination therapy in Alzheimer disease. Alzheimer Dis Assoc Disord 2008;22:209-21.

[18] Rountree SD, Chan W, Pavlik VN, Darby EJ, Siddiqui S, Doody RS. Persistent treatment with cholinesterase inhibitors and/or memantine slows clinical progression of Alzheimer disease. Alzheimers Res Ther 2009;1:7.

[19] Lopez OL, Becker JT, Wahed AS, Saxton J, Sweet RA, Wolk DA, et al. Long-term effects of the concomitant use of memantine with cholinesterase inhibition in Alzheimer disease. J Neurol Neurosurg Psychiatry 2009;80:600-7.

[20] Ott BR, Lapane KL. Tacrine therapy is associated with reduced mortality in nursing home residents with dementia. J Am Geriatr Soc 2002;50:35-40

[21] Lopez OL, Becker JT, Saxton J, Sweet RA, Klunk W, DeKosky ST. Alteration of a clinically meaningful outcome in the natural history of Alzheimer's disease by cholinesterase inhibition. J Am Geriatr Soc 2005;53:83-7.

[22] Geldmacher DS. Donepezil (Aricept) for treatment of Alzheimer's disease and other dementing conditions. Expert Rev Neurother 2004; 4:5-16.

[23] Zhu CW, Livote EE, Kahle-Wrobleski K, Scarmeas N, Albert M, Brandt $\mathrm{J}$, et al. Longitudinal medication usage in Alzheimer disease patients. Alzheimer Dis Assoc Disord 2010.
[24] Stern Y, Folstein M, Albert M, Richards M, Miller L, Bylsma F, et al. Multicenter study of predictors of disease course in Alzheimer disease (the "Predictors Study"): I. Study design, cohort description, and intersite comparisons. Alzheimer Dis Assoc Disord 1993;7:3-21.

[25] Richards M, Folstein M, Albert M, Miller L, Bylsma F, Lafleche G, et al. Multicenter study of predictors of disease course in Alzheimer disease (the "Predictors Study"): II. Neurological, psychiatric, and demographic influences on baseline measures of disease severity. Alzheimer Dis Assoc Disord 1993;7:22-32.

[26] Scarmeas N, Hadjigeorgiou GM, Papadimitriou A, Dubois B, Sarazin M, Brandt J, et al. Motor signs during the course of Alzheimer disease. Neurology 2004;63:975-82.

[27] Folstein MF, Folstein SE, McHugh PR. "Mini-mental state": a practical method for grading the cognitive state of patients for the clinician. J Psychiatry Res 1975;12:189-98.

[28] Stern Y, Sano M, Paulson J, Mayeux R. Modified Mini-Mental State Examination: validity and reliability. Neurology 1987;37:179.

[29] Devanand DP, Miller L, Richards M, Marder K, Bell K, Mayeux R, et al. The Columbia University Scale for Psychopathology in Alzheimer's Disease. Arch Neurol 1992;49:371-6.

[30] Stern MB, Hurting HI. The clinical characteristics of Parkinson's disease and parkinsonian syndromes: diagnosis and assessment. In: The Comprehensive Management of Parkinson's Disease. New York: PMA Corporation; 1978. p. 3-50.

[31] Richards M, Marder K, Bell K, Dooneief G, Mayeux R, Stern Y. Interrater reliability of extrapyramidal signs in a group assessed for dementia. Arch Neurol 1991;48:1147-9.

[32] Charlson ME, Pompei P, Ales KL, MacKenzie CR. A new method of classifying prognostic comorbidity in longitudinal studies: development and validation. J Chronic Dis 1987;40:373-83.

[33] Allison P. Event history analysis: regression for longitudinal event data. In: Sage University Paper Series on Quantitative Applications in the Social Sciences. Series no. 07-046. Beverly Hills, CA: Sage; 1984.

[34] Singer J, Willet J. Applied Longitudinal Data Analysis: Modeling Change and Event Occurrence. New York: Oxford University Press; 2003.

[35] Doody RS, Geldmacher DS, Gordon B, Perdomo CA, Pratt RD. Open-label, multicenter, phase 3 extension study of the safety and efficacy of donepezil in patients with Alzheimer disease. Arch Neurol 2001;58:427-33.

[36] Raskind MA, Peskind ER, Truyen L, Kershaw P, Damaraju CV. The cognitive benefits of galantamine are sustained for at least 36 months: a long-term extension trial. Arch Neurol 2004;61:252-6.

[37] Farlow MR, Lilly ML. Rivastigmine: an open-label, observational study of safety and effectiveness in treating patients with Alzheimer's disease for up to 5 years. BMC Geriatr 2005;5:3.

[38] D'Agostino RB, Lee ML, Belanger AJ, Cupples LA, Anderson K, Kannel WB. Relation of pooled logistic regression to time dependent Cox regression analysis: the Framingham Heart Study. Stat Med 1990; 9:1501-15. 